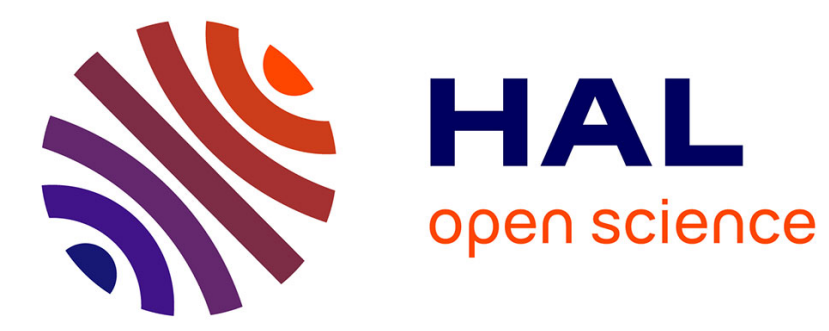

\title{
Multiphysical modeling of third-body rheology
}

Mathieu Renouf, Hong-Phong Cao, Viet-Hung Nhu

\section{To cite this version:}

Mathieu Renouf, Hong-Phong Cao, Viet-Hung Nhu. Multiphysical modeling of third-body rheology.

Tribology International, 2011, 44 (4), pp.417-425. hal-00871153

\section{HAL Id: hal-00871153 \\ https://hal.science/hal-00871153}

Submitted on 8 Oct 2013

HAL is a multi-disciplinary open access archive for the deposit and dissemination of scientific research documents, whether they are published or not. The documents may come from teaching and research institutions in France or abroad, or from public or private research centers.
L'archive ouverte pluridisciplinaire HAL, est destinée au dépôt et à la diffusion de documents scientifiques de niveau recherche, publiés ou non, émanant des établissements d'enseignement et de recherche français ou étrangers, des laboratoires publics ou privés. 


\author{
Multiphysical modeling of third-body rheology \\ M. Renouf, H.-P. Cao and V.-H. Nhu \\ Université Lyon, CNRS \\ LaMCoS, INSA-Lyon UMR5259, F-69621 Villeurbanne cedex, France
}

\begin{abstract}
As the rheology of the third body does not only depend on its mechanical properties, classical discrete element simulations are not capable of modeling its flows. Consequently to take into account the third body's mechanical, thermal and physicochemical properties, an extended discrete element approach is proposed and applied to the simulation of third body flows. Each extension of the standard DEM model is compared to experimental results. The extended model's efficiency is demonstrated by using an arbitrary physicochemical law that simulates different types of behaviour observed experimentally.
\end{abstract}

Key words: third body, DEM, thermal, physico-chemical

\title{
Nomenclature
}

$a$ Contact radius

c Specific heat

$\mathcal{E}^{\text {diss }}$ Dissipated energy

$E *$ Effective Young modulus

$\mathbf{F}^{e x t}$ External forces 
$g$ Distance between particles

$\mathbb{H}$ Linear mapping from contact to body level

$H$ Contact conductance

$h$ Time step

$k_{t h}$ Contact thermal conductivity

M Mass matrix

$Q_{i}^{\text {cond }}$ Thermal power due to conduction

$Q_{i}^{\text {diss }}$ Dissipated mechanical power

q Configuration parameter

$\dot{\mathbf{q}}$ First time derivative of the configuration parameter

d $\dot{\mathbf{q}}$ Differential measurement of acceleration

$\mathbf{R}$ Contact forces

$R *$ Effective radius

r Local contact impulse

$r$ Particle radius

$T$ Particle temperature

$t$ time variable

v Local relative velocity 
$\mathbb{W}$ Delassus operator

Greek symbols

$\gamma$ Local cohesion

$\eta$ Surface energy

$\mu$ Global friction coefficient

$\rho$ Density

$\theta$ Ponderation of time integrator

Subscripts

free without contact forces

$i, j$ particle

$n$ normal component in the local frame

$t$ tangential component in the local frame

$\alpha$ contact

Superscripts

* transpose

' effective quantities 


\section{INTRODUCTION}

The well-known concept of the third body was introduced by Godet in the seventies [1] to characterize the discontinuous and heterogeneous interface that separates two bodies in contact. This thin layer (ranging from several nanometers to several micrometers in thickness) appears to possess its own rheology that depends on contact conditions, material properties and often additional unknown parameters. Although it is mainly linked to essentially mechanical aspects, such as velocity accommodation [2], load carrying capacity [3] and solid lubrication [4], it plays an important role in other physical aspects. For example, it ensures the thermal continuity between two bodies in contact and explains the gap in temperature observed experimentally $[5,6]$. Moreover, it is able to sustain the maximal contact temperature within its thickness [7] and, when considering electrical aspects, it can play the role of electrical capacity, as it acts as an insulator with respect to external conditions such as humidity [8].

Due to the difficulty of instrumenting a real contact without disturbing local rheology, observations of third body rheology can only be performed on simplified experimental set-ups. To reproduce and try to understand a real contact in the presence of the third body, numerical tools have been developed and adapted to meet the new challenge raised by this concept. The discontinuity and heterogeneity of such interfaces has led researchers to use discrete element methods (DEM) to describe its evolution. Based on the discrete element model proposed by Cundall [9], several authors have analysed the mechanical behaviour of the third body [10] and tried to characterize its

rheology as a function of local parameters [11], geometry conditions [12] and 
also proposed several DEM-based wear models [13]. Nevertheless in numerous cases, such modeling remains phenomenological and sometimes depends on the method itself. More recently the classical discrete element approach have been achieved to take into account weak coupling either between electrical and mechanical phenomena [14] or between thermal and mechanical phenomena [15]. The latter improvements represent a step forward in the multi physical modeling of the third body and provided interesting results. However, one important point is still missing which is that the physicochemical properties of the interface are neglected or considered independent of mechanical, thermal and electrical stresses. Nonetheless, variations of these parameters have significant effects on the mechanical responses of the interface $[16,17,18,19]$. Consequently, the next step in third body modeling cannot neglect physicochemical aspects or, in particular, their dependencies on external stresses.

In this context, the present paper proposes a unified discrete element approach capable of handling the multi-physical modeling of discrete assemblies. Mechanical, thermal and physicochemical aspects are considered as well as their interactions. Thus Section 2 presents the basic principles of the mechanical formulation used for the present work. The thermal formulation is presented in Section 3 while the relations between the thermal, mechanical and physicochemical properties of the third body are treated in Section 4. Section 5 is dedicated to the numerical results from third body flow simulations and Section 6 concludes the paper. 


\section{Mechanical modeling}

\subsection{Background}

In the case where a medium is considered fully discontinuous, Discrete Element Methods (DEM) appear well-suited for describing these phenomena. In the case where the problem cannot be described by continuum mechanics, it appears more interesting to represent the medium concerned as initially discontinuous and compute the evolution of each element. Moreover, it is important to take this discontinuity into account when the heterogeneity of the medium has a strong influence on its evolution (which is the case in tribological problems).

The development of DEM started with the pioneering works of Cundall who developed the Distinct Element Method [9]. Initially used to simulate rock systems, the method was then extended to the simulation of granular media [20]. Contact interactions are described by a compliant model related to an admissible numerical penetration. Then the so-called Granular Element Method [21] appeared as an improvement of the method. An incremental formulation, an iterative process and a convergence criterion are added to respect the balance equation that was not always satisfied in the initial method. GEM has been used for studying different plasticity deformation modes [22].

Another contribution to DEMs was a variant of the Cundall approach known as Molecular Dynamics (MD) [23], which consists in simulating the dynamics of atoms and molecules in order to deduce the macroscopic properties of the material studied. Like the Cundall method and GEM, MD makes use of a compliant model to describe contact between particles. The balance 
equations are not always satisfied but the simulation time step is kept small enough to ensure the stability of the integration scheme. Moreover, it is possible to add numerical artefacts, such as numerical viscosity to control the evolution of energy in the system.

The Contact Dynamics method, initially developed by Moreau [24] and based on the convex analysis framework, appears to be a different approach. Contrary to compliant models, no regularization scheme is used to describe particle interactions: the non smooth contact feature is preserved by way of an implicit formulation of the global contact problem solved classically by using a projected block splitting algorithm [25]. Further works led to the extension of the method to multi-contact simulations of collections of deformable bodies [26] and the method became the so-called Non Smooth Contact Dynamics method (NSCD).

The first utilization of DEM in tribology and more specifically for modeling third body flows dates back to the nineties with the works of Elrod and Brewe [10]. These works, based on Cundall's approach, were exploratory and led many authors to try such models to investigate the influence of numerical parameters [11] or the influence of particle size [12] on the mechanical response of media. Later, certain authors used this approach to identify the influence of grain size in shear simulations, using a JKR model with a constant coefficient [27] and leading to unclear conclusions regarding the effect of size and cohesion. Of more interest, Fillot et al proposed to characterize wear mechanisms $[13,28]$ by developing several DEM-based wear laws. Nevertheless, this dimensionless study was performed using interconnected parameters leading to specific rather than generic results. Recently, several 
authors have proposed using deformable particles instead of rigid particles in third body flows $[29,30]$. The first author proposed mixing discrete and continuous formulations to take into account the effect of the first body on third body rheology while the second used deformable particles to simulate third body flows. Such approaches underline the importance of extending classical approaches in order to to make progress in the simulation of third body flows.

\subsection{Mechanical formulation}

The mechanical approach used in the present paper stems from the Contact Dynamics framework developed by Moreau [24] and extended by Jean [26]. This approach has shown its relevancy for modeling the evolution of discontinuous media in numerous cases $[31,32,33,14]$. An overview is proposed in this section and the reader can refer to the original works to obtain further details.

A large number of shocks can be expected in dense discontinuous systems. Velocity may be discontinuous and acceleration cannot be defined as the usual second time derivative of the configuration parameter $\mathbf{q}$. If the time step of the simulation is not small enough, numerical instabilities may appear and disturb the physical results. Consequently, the classical equation of motion is reformulated in the terms of an equation of differential measurement:

$$
\mathbb{M} d \dot{\mathbf{q}}=\mathbf{F}^{e x t}(t, \mathbf{q}, \dot{\mathbf{q}}) d t+d \mathbf{R},
$$

where $\mathbb{M}$ represents the inertia matrix and $\mathbf{F}^{e x t}$ the external forces. $d t$ is the Lebesgue measurement in the real space $\mathbb{R}, d \dot{\mathbf{q}}$ is a differential measurement 
representing the acceleration and $d \mathbf{R}$ is a differential measurement of the impulses.

As the number of contacts is larger than the number of bodies, local variables defined in local frames (contacts) are preferred to global ones (bodies) i.e. local relative velocities $\mathbf{v}_{\alpha}$ ( $\alpha$, index of the contact number) are concatenated in a vector $\mathbf{v}$, the local impulses $\mathbf{r}_{\alpha}$ in a vector $\mathbf{r}$. The corresponding global variables are the generalized velocities of the system (collection of bodies) $\dot{\mathbf{q}}$, the first time derivative of the configuration parameter $\mathbf{q}$, and the contact forces $\mathbf{R}$ applied to all the bodies. Local variables are related to global variables via the linear mapping $\mathbb{H}$ that transfers information computed at the contact points to the bodies in contact. This can be summarized by the following system:

$$
\left\{\begin{array}{l}
\mathbf{R}=\mathbb{H} \mathbf{r} \\
\mathbf{v}=\mathbb{H}^{*} \dot{\mathbf{q}}
\end{array},\right.
$$

where $\mathbb{H}^{*}$ is the transpose of $\mathbb{H}$. Thus both mappings contain all the local information, such as the local frame defined at each contact point and the network connectivity of each contact.

A $\theta$ integration scheme is used to discretize Equation (1). The scheme stability condition implies that $\theta$ remains between $1 / 2$ and 1 . The $\theta$-method is an implicit scheme, equal to the backward Euler scheme when $\theta$ is equal to 1 . Then, proceeding to the time discretization, the contact problem is solved over interval $] t, t+h]$ of length $h$ in the terms defined previously. Successive approximations of both Equation (1) and the first time derivative 
of the configuration parameter lead to the following system:

$$
\left\{\begin{array}{l}
\dot{\mathbf{q}}(t+h)=\dot{\mathbf{q}}^{\text {free }}(t)+h\left(\mathbb{M}^{-1}\right) \mathbf{R}(t+h) \\
\mathbf{q}(t+h)=\mathbf{q}(t)+h \theta \dot{\mathbf{q}}(t+h)+h(1-\theta) \dot{\mathbf{q}}(t)
\end{array}\right.
$$

with

$$
\dot{\mathbf{q}}^{\text {free }}(t)=\dot{\mathbf{q}}(t)+\mathbb{M}^{-1} h\left(\theta \mathbf{F}^{e x t}(t+h)+(1-\theta) \mathbf{F}^{e x t}(t)\right)
$$

where $\dot{\mathbf{q}}^{\text {free }}$ denotes the free velocity (velocity computed without contact forces). For rigid body systems, the inertia matrix $\mathbb{M}$ is diagonal and easily invertible, the internal forces vanish and the external forces are given by a function of time.

The second equation of System (3) is used to update it. When $\theta$ is equal to 1 , contact detection is performed using the configuration parameter at time $t_{i}$. On the contrary, when $\theta \neq 1$, contact detection is performed using a prediction of the configuration parameter (at time $t_{i}+h(1-\theta)$ ). In this case the $\theta$-method corresponds to the well-known leap-frog scheme: The contact point and the local frame are computed in this temporary position which is assumed to be closer to the solution than the position at time $t_{i}$.

Using equations (2) in the first equation of System (3), the global discretization of the equation of motion and the contact laws can be summarized in the following system:

$$
\left\{\begin{array}{l}
\mathbb{W} h \mathbf{r}(t+h)-\mathbf{v}(t+h)=-\mathbf{v}^{\text {free }} \\
\text { ContactLaw }[\mathbf{v}(t+h), \mathbf{r}(t+h)]
\end{array}\right.
$$

where $\mathbb{W}\left(=\mathbb{H}^{*} \mathbb{M}^{-1} \mathbb{H}\right)$ is the Delassus operator that models the local behaviour of the solids at the contact points. The right-hand-side of the first equation of System (4) represents the free relative velocity that accounts only 
for the internal and external forces $\mathbf{F}(t)$. The second equation of System (4) requires that the contact law must be satisfied by each component of the couple $(\mathbf{v}(t+h), \mathbf{r}(t+h))$. To solve system (4), the so-called Block Non Smooth Gauss-Seidel algorithm (NSGS) is used [26]. It has proven to be very robust and efficient for a large number of heterogeneous problems [31, 32] and it draws advantage from a parallel version [34] to ensure reduced simulation time.

\subsection{Interaction laws}

When collections of rigid bodies are considered, the physical behaviour of the system is highly dependent on the particle interaction law. As body deformations are not taken into account, contact laws control the evolution and rheology of the media and must be chosen according to the behaviour of the real medium. Consequently, a specific law with a specific set of parameters can only characterize a specific type of third body and numerical parameters must be defined using experimental data.

In the present context, the main purpose is not to accurately define the contact law, but to propose and illustrate a multi-physical approach. The latter is intended to be generic and adaptable to any local third body behaviour. For the purposes of this work, a non-smooth cohesive contact law (Figure 1a) is used. It could be seen as the non-smooth extension of a force which derives from a Lennard-Jones potential. Such a law has been used in the case of a wheel-rail contact [8, 14], focusing on metal powder.

Two parameters are considered: A cohesive force $\gamma$ that represents a constant force in opposition to body detachment and a distance $d_{w}$ that defines the attraction area of each body. The chart of the non-smooth cohesive force 

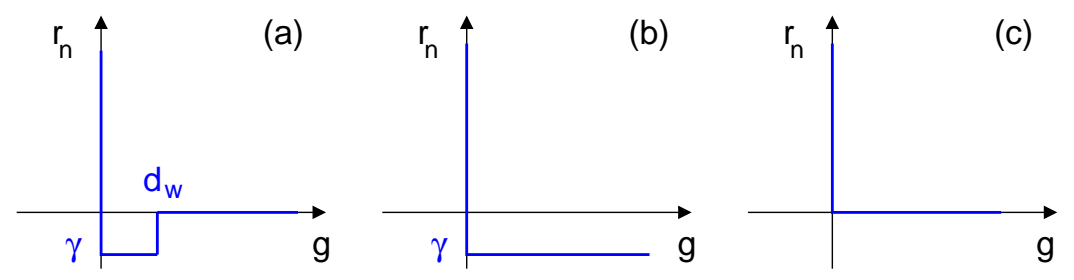

Figure 1: Chart of the non-smooth cohesive force (a) as a combination of two Signorini charts with (b) and without (c) a $\gamma$-translation along the $r_{n}$ axis. $\gamma$ represents the cohesive force while $g$ is the distance between bodies and $r_{n}$ is the normal component of the contact force

can be seen as a combination of two Signorini charts as a function of contact status (Figure 1b and 1c). If body attraction areas do not overlap, then the contact status is non contact $\left(r_{n}=0\right.$ and $\left.g>d_{w}\right)$. If body attraction areas overlap and the gap between bodies is not equal to zero, interference by an additional cohesive force occurs. Therefore the contact status is $\mathrm{co}$ hesive $\left(\gamma<r_{n}<0\right.$ and $\left.g \leq d_{w}\right)$. Finally the contact status is stick when the gap vanishes $\left(r_{n} \geq 0\right.$ and $\left.g=0\right)$. When $\gamma=0$ then $d_{w}=0$ and the cohesive status is no longer considered. In this case the contact description is the classical Signorini condition used to simulate dry systems [26]. The non contact condition appears as a condition of non attraction between the candidate and antagonist bodies.

\section{Thermal formulation}

\subsection{Background}

Different heat transfer mechanisms have been identified in discrete, discontinuous and porous media, i.e. conduction through the particles and the 
contacts, conduction and convection through the interstitial medium, and radiation between the particles. When the behaviour of a solid third body is investigated (for example, metallic powders with an interstitial medium such as air), the thermal conductivity of such a gas is very low compared to that of the material. Consequently, it can be assumed that convection (free and forced) as well as conduction through the medium are negligible [35]. Moreover, the temperature considered herein ranges from $0 \mathrm{~K}$ to 500 K. Therefore radiant heat transfer through small pores (around $1 \mu \mathrm{m}$ ) can also be neglected [36]. Thus only conduction through the solids and the contact areas are considered in the present work as a heat transfer process. Nevertheless, another thermal process must be taken into account, i.e. local heat generation due to mechanical transformations and stresses. The latter process is the main cause of flash temperatures in the contact [6].

From a numerical viewpoint, coupling the dynamical evolution of discrete element assemblies and thermal effects was reported in the $\mathrm{PhD}$ thesis by Vargas-Escobar [37] who defined the concept of Thermal Particle Dynamics (TPD). Conduction is considered to transfer heat from certain sources to the whole medium, although the relative motion between particles does not generate heat. Consequently, shear simulations do not increase the temperature of the medium and TPD cannot be used to model a "real contact" with a solid third body. As an extension of the previous approach, some authors proposed to transform the mechanical energy dissipated in heat in the contact. To achieve this, Richard et al [15] used normal contact dissipation while neglecting tangential contact dissipation, contrary to Nguyen et al [38] who did quite the reverse. In both cases, only one part of the 
mechanical dissipation is taken into account and relies strongly on numerical parameters. Nevertheless such approaches appear to constitute the first step towards coupling mechanical and thermal effects and propose pioneering numerical investigations into the thermal mechanical behaviour of discrete contact interfaces.

\subsection{Contact conductance}

Heat transfer by conduction between two particles in contact mainly depends on particle surface roughness that imposes the way in which heat flow lines pass through the contact area. When developing models to investigate the third body, the order of magnitude of particle radii ranges from one hundred nanometers to several micrometers (according to the accuracy of the description). In this case, it can be assumed that the influence of surface roughness is negligible and the heat flux through the contact can be expressed as a function of the contact area [39]. Thus the contact conductance between two particles can be obtained by:

$$
Q_{i j}^{c o n d}=H\left(T_{i}-T_{j}\right)
$$

where $\mathrm{H}$ is the contact conductance that depends on the contact area and thermal conductivity of the particles involved. This formula is commonly used to compute the heat flux between two spheres [35, 40]. Thus, based on the Hertz theory, for three-dimensional modeling contact conductance is expressed by:

$$
H_{3 D}=2 k_{t h} a=2 k_{t h}\left(\frac{3 r_{n} R^{\prime}}{4 E^{\prime}}\right)^{1 / 3}
$$


$E^{\prime}$ represents the effective Young Modulus of the particles, $R^{\prime}$ is the effective radius and $a$ is the contact radius. $r_{n}$ denotes the normal forces defined in Section 2. $k_{t h}$ denotes the contact thermal conductivity. In the case of two-dimensional modeling, Equation (6) could not be used and must be adapted from the work of Yovanovich [39] to express contact conductance $\mathrm{H}$ as a function of the contact force between two cylinders. In this case, the following is obtained:

$$
H_{2 D}=2 k_{t h} \sqrt{2 a L}=2 k_{t h}\left(\frac{8 r_{n} R^{\prime}}{\pi L E^{\prime}}\right)^{1 / 4} .
$$

where $\mathrm{L}$ is the length of the cylinder, equal to unity in a two dimensionnal model.

\subsection{Local energy dissipation}

Within discontinuous media, we assume that relative particle motion can generate heat. This assumption is backed by experiments on sapphire/steel contact [6] underlining increased temperature in the presence of the third body. From a numerical viewpoint, dissipation of mechanical energy primarily depends on the contact law between the particles. If local relative motions are considered, then only dissipative processes should be taken into account.

In the case of the law defined in section 2.3, there is no elastic deformation. Thus the variation of local kinetic energy due to a collision or a sliding contact is transformed into heat and splits between contacting particles. For a single particle, the dissipated energy is equal to:

$$
\mathcal{E}^{\text {diss }}=\frac{1}{2} \mathbb{M}\left(\dot{\mathbf{q}}(t+h)^{2}-\dot{\mathbf{q}}(t)^{2}\right),
$$


where $\mathbb{M}$ is the inertia matrix, and $\mathbf{q}(t)$ and $\dot{\mathbf{q}}(t+h)$ are the velocity vectors before and after the event. According to equation (1), it is possible to write:

$$
\mathbb{M}(\dot{\mathbf{q}}(t+h)-\dot{\mathbf{q}}(t))=\mathbf{R},
$$

where $\mathbf{R}$ is the contact vector. Thus the dissipated energy can be written as:

$$
\mathcal{E}^{\text {diss }}=\mathbf{R} \frac{(\dot{\mathbf{q}}(t+h)+\dot{\mathbf{q}}(t+h))}{2}
$$

and can be converted into a heat flux on the time interval $[t, t+h]$ :

$$
Q_{i j}^{d i s s}=\mathbf{R} \frac{(\dot{\mathbf{q}}(t+h)+\dot{\mathbf{q}}(t))}{2 d t},
$$

where $d t$ is the time step used to discretize the evolving temperature.

\subsection{Global formulation}

To describe the evolution of the temperature of a single particle, the scheme used for the mechanical evolution is also used ( $\theta$ integrator) where $\theta$ is taken as being equal to 0.5 to obtain an unconditionally stable scheme. Thus the temperature is given by:

$$
T_{i}(t+d t)=T_{i}(t)+\theta \dot{T}_{i}(t+d t) d t+(1-\theta) \dot{T}_{i}(t) d t
$$

where term $\dot{T}_{i}(t+d t)$ represents the time derivative of the temperature and is equal to:

$$
\dot{T}_{i}(t+d t)=\frac{1}{\rho_{i} C_{i} V_{i}}\left(Q_{i}^{\text {cond }}+Q_{i}^{\text {diss }}\right) .
$$

In Equation (13), the term " $\rho_{i} C_{i} V_{i}$ " represents the thermal capacity of the particle concerned. $Q_{i}^{\text {cond }}$ represents the sum of the thermal power due to the 
conduction between the particle $i$ and its neighbourhood given by Equation (5). $Q_{i}^{\text {diss }}$ denotes the mechanical power dissipated by particle $i$ due to its relative motion with neighbouring particles given by Equation (11). The validity of Equation (13) is based on the fact that contact conductance should be negligible compared to the particle conductance of the material. This assumption results in a maximal value for the simulation time step [35], that is generally less than the condition given by mechanical assumptions [15].

\section{Physicochemistry of the interface}

The physicochemical properties of the interface play an important role in its rheology and, consequently, in the mechanical response [16]. Moreover, such properties strongly depend on mechanical and thermal stresses [5, 6]. Thus when both aspects are considered it is important to account for such dependency in the model selected.

Firstly, we define the cohesion force $\gamma$ acting between the particles used in the contact law (c.f. Subsection 2.3). According to the pioneering work of Johnson et al [41], this cohesive force depends on their surface energy and radius. Consequently, a simple model, a function of particle radius and surface energy, is used to define the cohesive force:

$$
\gamma=2 \frac{\eta_{i} \eta_{j}}{\eta_{i} r_{j}+\eta_{j} r_{i}}
$$

where $\eta_{i}$ and $\eta_{j}$ denote the surface energy of the particles $i$ and $j$ respectively while $r_{\text {eff }}$ represents the effective radius. Equation (14) causes the cohesion value to vanish when the surface energy of one particle is equal to zero. 
Although cohesion forces depend on surface energy according to several assumptions [42, 43], surface energy depends on particle temperature. Such suppositions have been highlighted by experiments performed on different types of material $[17,6,19]$. In order to account for this dependency, the surface energy $\eta$ of each particle is defined as follows:

$$
\eta=\eta_{i n i}+\eta_{v a r} \cdot \tanh \left(\frac{T_{t r}-T}{T_{v a r}}\right)
$$

where $\eta_{i n i}$ is the mean surface energy and $\eta_{v a r}$ the magnitude of its variation. $T_{v a r}$ is the temperature interval over which the transition occurs while $T_{t r}$ is a threshold value for the temperature. Equation (15) is purely arbitrary and must be obtained from experimental or theoretical data when a specific material is investigated. Nevertheless, this type of model allows characterizing several kinds of behaviours: It could model the surface energy $\eta$ as an increasing function or a decreasing function of the temperature (according to the sign of $\left.\eta_{v a r}\right)$. As Equation (15) represents the behaviour of the material, results will be sensitive to the present law.

\section{Simulation results}

\subsection{Numerical set-up}

To illustrate the efficiency of the method, the interface is represented by a set of 4800 rigid spherical particles free to move between upper and lower boundaries composed of rigid particles. The radius of free particles and boundary particles range from 0.8 to $1.2 \mu \mathrm{m}$. The upper wall is free to move only in the Z-direction and is subjected to a vertical force. The lower 
wall moves only in the X-direction with a constant speed. Periodic conditions are used in the $\mathrm{X}$ and $\mathrm{Y}$-directions to simulate an infinite contact.

In addition to classical boundary conditions, a thermal condition is applied on the upper and lower boundaries of classical mechanical boundary conditions to avoid adiabatic effects, as performed by Richard et al [15]. Both boundaries are considered with a given thickness several orders larger than the thickness of the sample. Thus heat conduction across both boundaries is written using the following two-dimensional heat equation:

$$
k\left(\frac{\partial^{2} T}{\partial z^{2}}+\frac{\partial^{2} T}{\partial x^{2}}\right)=\rho c \frac{\partial T}{\partial t}
$$

where $k, \rho$ and $c$ denote the thermal conductivity, density and specific heat, respectively, of the boundary considered.
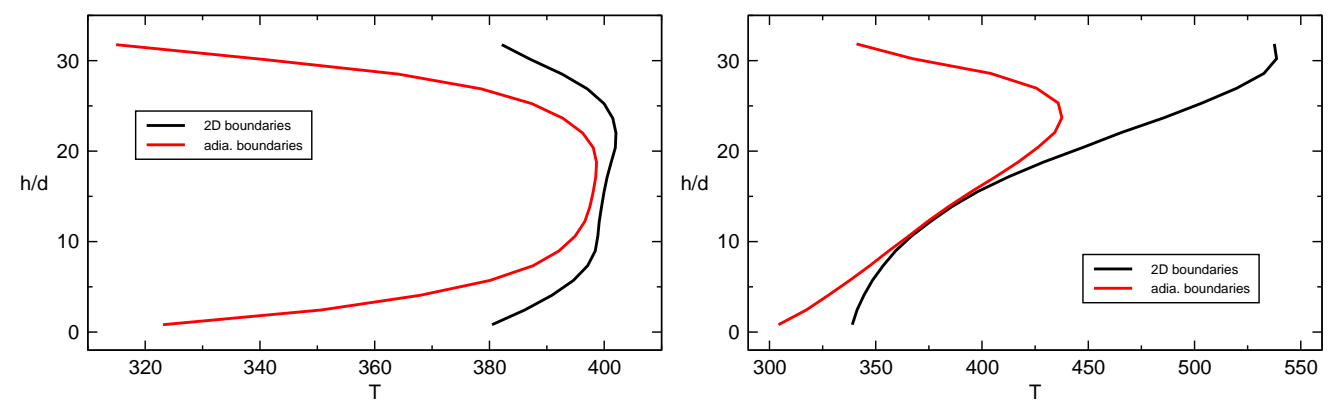

Figure 2: Influence of the thermal boundary condition on the temperature profile through the thickness of the sample: $\gamma=0$ (Left) and $\gamma=0.05$ (Right)

At each time step the temperature of the sample close to the boundaries acts as a limit condition. As the boundary thickness is larger than the thickness of the sample, the latter assumption is always satisfied. Note that the two-dimensional heat equation is preferred to the one-dimensional heat 
equation to avoid an average effect close to the boundary. Such boundary conditions are strongly recommended for simulating non insulated shearing, as shown in Figure 2 which compares the temperature profile with and without thermal boundary conditions for different internal cohesion values.

\subsection{Thermal mechanical behaviour}

\subsubsection{Static case}

Before performing dynamical simulations to check the validity of the thermal mechanical model, two comparisons with experimental and previous numerical results were performed for static configurations. The first concerned the evolution of the temperature of a ball in a vertical column [44] while the second concerned the evolution of temperature in densely packed cylinders.

In the first case, the experimental apparatus of [44] was reproduced numerically: 15 spheres were stacked between two vertical parallel wood plates (see Figure 3a). A normal load of $160 \mathrm{~N}$ compressed the stack and the bottom of the pile was in contact with a heat source of $107 \mathrm{C}$.

The evolution of the temperature of the second sphere in the column was plotted in logarithm-logarithm representation for the simulation and the experimental result obtained in [44]. Figure 3b shows a good match between the two curves for different sphere in the column. Thus this first example is an initial validation of the numerical model. Note that the model takes into account the contact between spheres and between spheres and plates, which is of great importance when matching with experimental results.

The second example concerns a densely packed cylinder. A static twodimensional simulation was performed and compared to the benchmark proposed by Vargas [37]. In the present simulation, conduction is governed by 

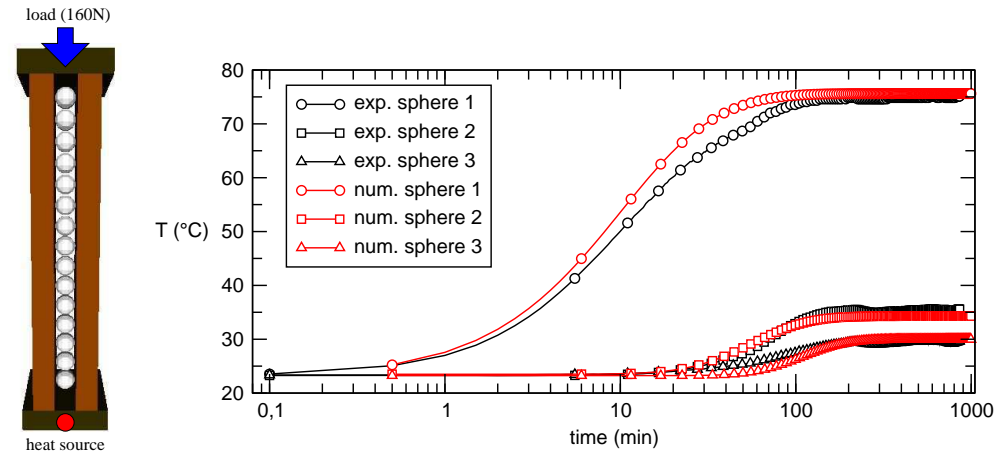

Figure 3: On the left: Numerical reproduction of the experiment by Yun et al [44]. On the right: the evolution of the temperature of different spheres in the column for the experiment and the simulation (sphere 1,4 and 7)

Equation (7) due to the two-dimensional nature of the simulation (cylinder are used). The results are compared to the three-dimensional simulation proposed in [37] using a conduction governed by Equation (6) valid only for spheres.

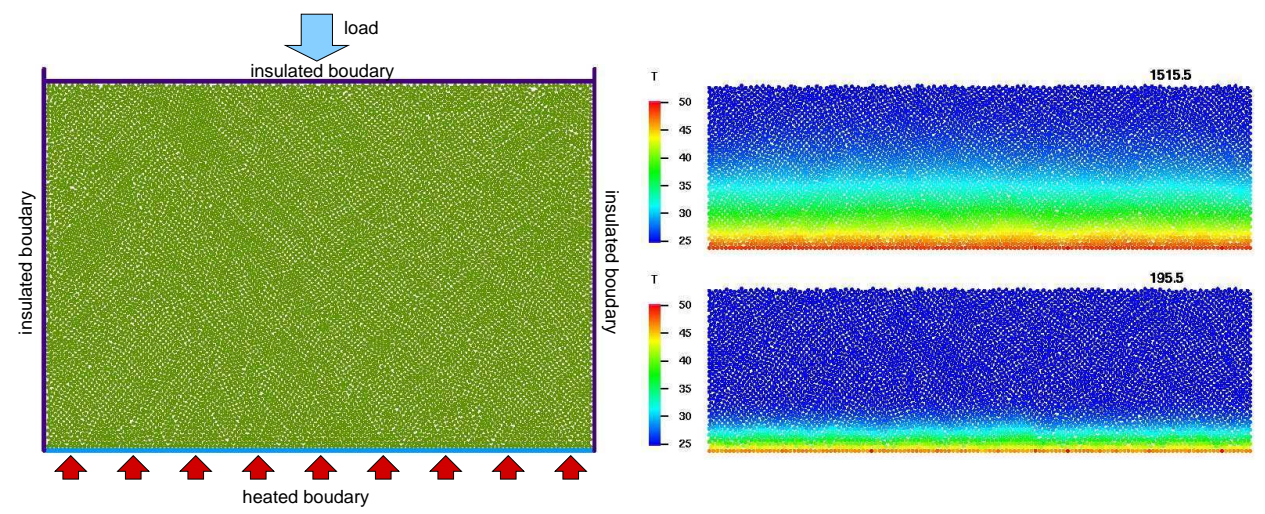

Figure 4: On the left: two-dimensional packing of disks subjected to a heat source and vertical compression. On the right: evolution of the temperature inside the packing for $t=3 \min$ and $t=25 \min$. 
The temperature profiles obtained with the two-dimensional simulation are similar to the profile obtained in [37]. Snapshots of the temperature field taken at two different moments $(t=3 \mathrm{~min}$ and $t=25 \mathrm{~min})$ are very similar in both cases. Consequently, the two-dimensional restriction of equation (6) gives a good correlation with three-dimensional simulations.

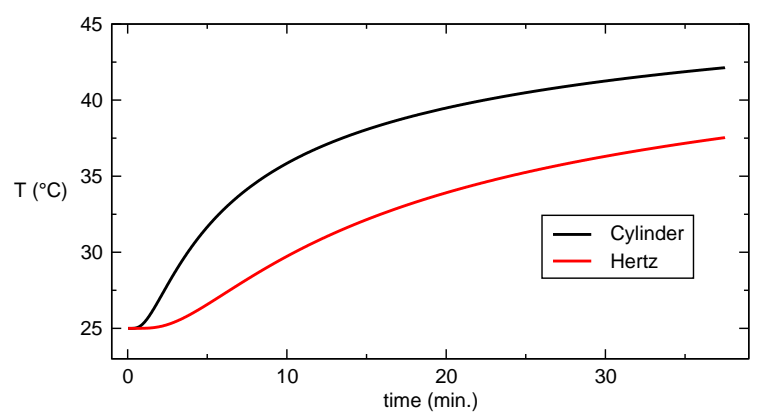

Figure 5: Evolution of the temperature within the packing of a cylinder for a conduction governed by Equation (6) (Hertz case) and Equation (7) (Cylinder case).

Figure 5 shows the evolution of the temperature of a cylinder in the packing using a conduction governed by Equation (6) (Hertz case) and Equation (7) (Cylinder case). The figure underlines the importance of using the twodimensional restriction in a two-dimensional simulation. If a conduction governed by Equation (6) is used in two-dimensions (as in [38] for example), errors are introduced in the temperature profile and the different temperatures measured in the sample.

\subsubsection{Dynamical case}

After the validation phase in static configuration, simple shear simulations were performed to investigate the evolution of the temperature profile through the thickness of the sample (c.f. figure 6). 


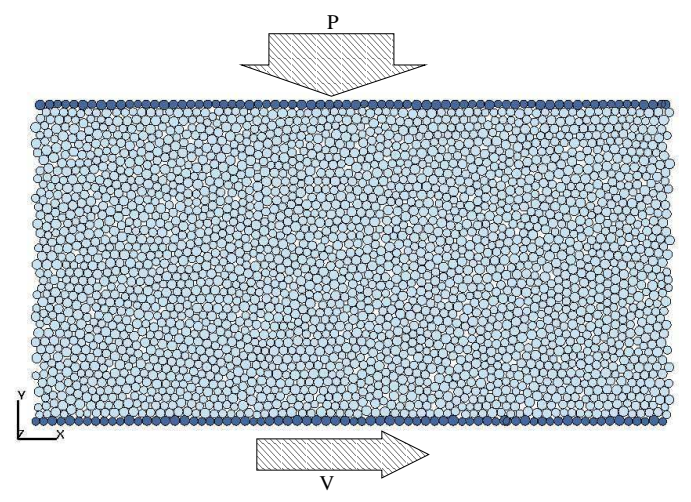

Figure 6: Simulation sample used for simple shear simulations

The two-dimensional sample is composed of 3000 rigid particles. Their radius range from $0.1210^{-6} \mathrm{~m}$ to $0.1610^{-6} \mathrm{~m}$. The high and the length of the sample are respectively equal to $1010^{-3} \mathrm{~m}$ and $2010^{-3} \mathrm{~m}$. A vertical force equal to $1 N$ compresses the sample, while a tangential velocity equal to $5 \mathrm{~m} . \mathrm{s}^{-1}$ shears the sample. These simulations were performed using different internal cohesion values ( $\gamma$ parameter). Each profile results from an average computation in steady state.
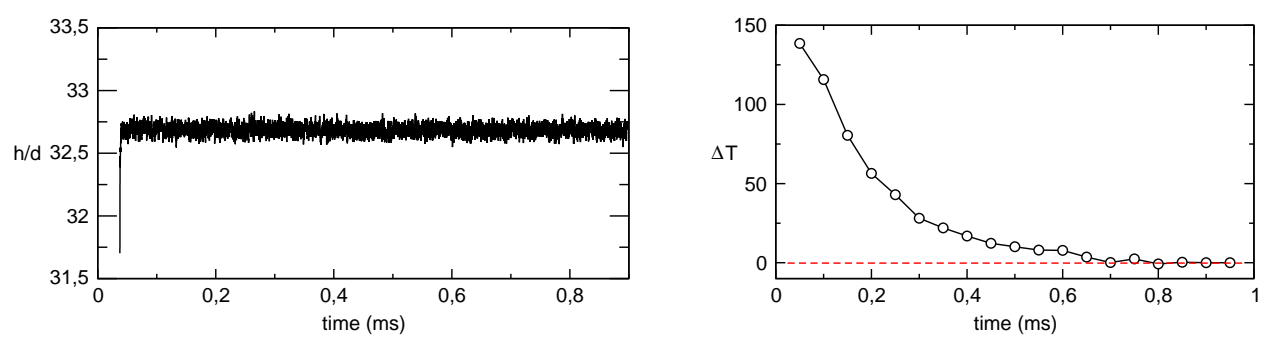

Figure 7: Indicators of steady state for the computation of velocity and temperature profile. The evolution of the position of the upper plate can be seen on the left while the evolution of the maximal temperature gradient can be seen on the right. 
Regarding the velocity profile through the thickness, the steady regime is reached when the upper plate has a quasi constant position (see left part of figure 7 ), whereas for the temperature profile through the thickness, steady state is reached when the temperature gradient is close to zero (see right part of figure 7 ).
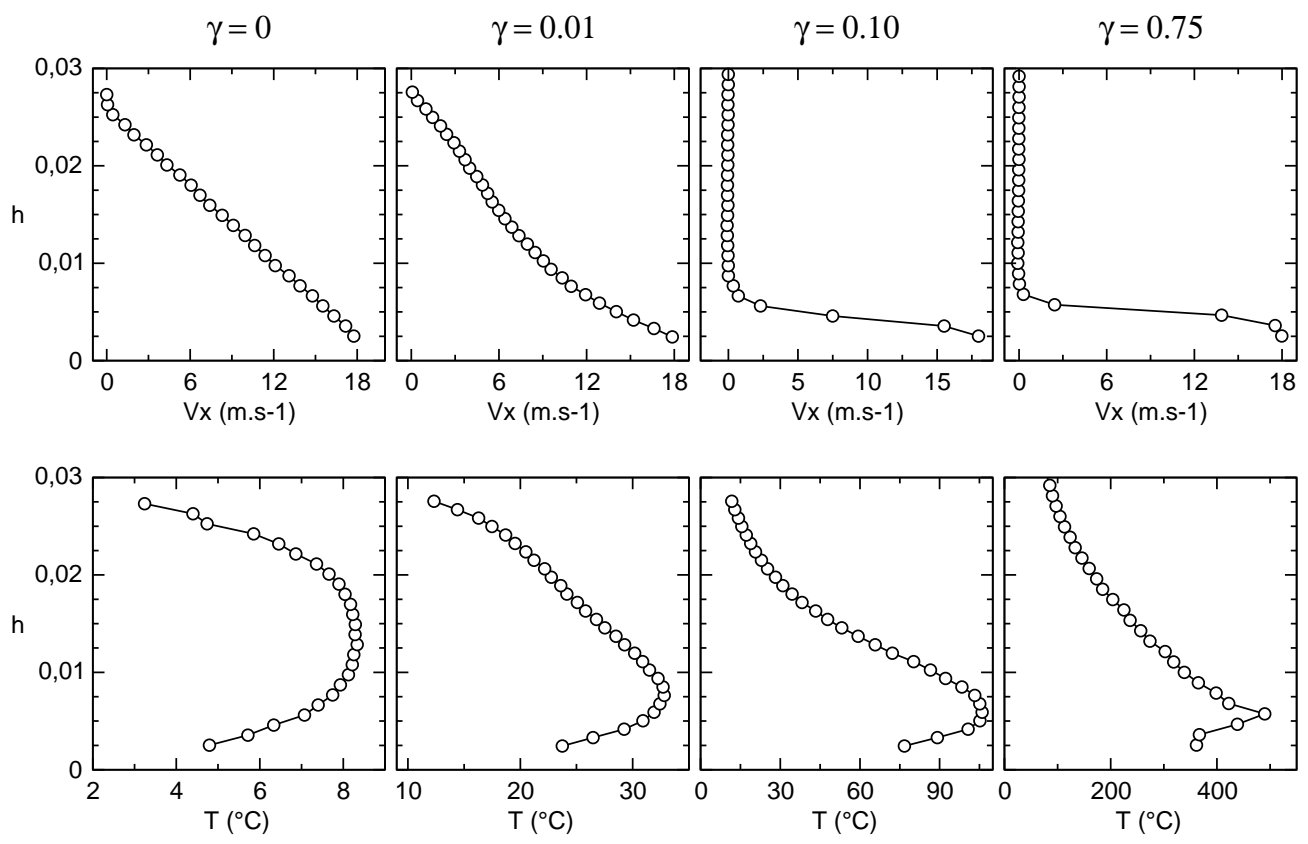

Figure 8: Shear velocity profiles (up) and temperature profiles (down) through the sample thickness for different cohesion values

The velocity and temperature profiles through the sample thickness are represented on figure 8 for different cohesion values. Localization occurs near the moving boundary when the sample cohesion increases. The thickness of the shear band decreases as cohesion increases and remains the locus of the maximal temperature value. Moreover the temperature of the boundaries is no longer equal as observed in a previous thermal study [15]. 


\subsection{Thermal physicochemical mechanical behaviour}

The complete model which couples mechanical, thermal and physicochemical effects is finally tested in a simple shear simulation. The following law is used to couple the surface energy to the temperature:

$$
\eta=0.075+0.05 \cdot \tanh \left(\frac{800-T}{10}\right) .
$$

Equation (17) describes a decrease of surface energy $\eta$ with temperature. The energy remains constant for a temperature lower than $790(\eta=0.125)$. Then the decrease takes place on the interval $[790,810]$ to reach a new constant value after $810(\eta=0.025)$. Consequently, the cohesion in the sample varies from a high value $(0.125)$ to a low value $(\gamma=0.025)$. Simulations are performed with the law given above and snapshots are presented in figure 9 .

During the first steps of the simulation, the temperature increases throughout the medium with localization of the maximal value close to the upper wall as obtained with a constant cohesion value. During this phase, all the particles in the sample are affected by the given shear velocity. Then, when the temperature reaches the temperature threshold given by equation (17), the surface energy value decreases at the locus of the maximal temperature and creates a shear band. Then, once the shear band forms, its thickness increases slowly and accommodates the shearing process.

This localization has consequences on the evolution of the friction coefficient as well as the evolution of the temperature within the sample. First, the global friction coefficient decreases from a value of 0.315 to a value of 0.167 (Left of figure 10) which correspond to the friction values obtained with a high $(\gamma=0.125)$ and a low cohesion value $(\gamma=0.025)$, respectively. 


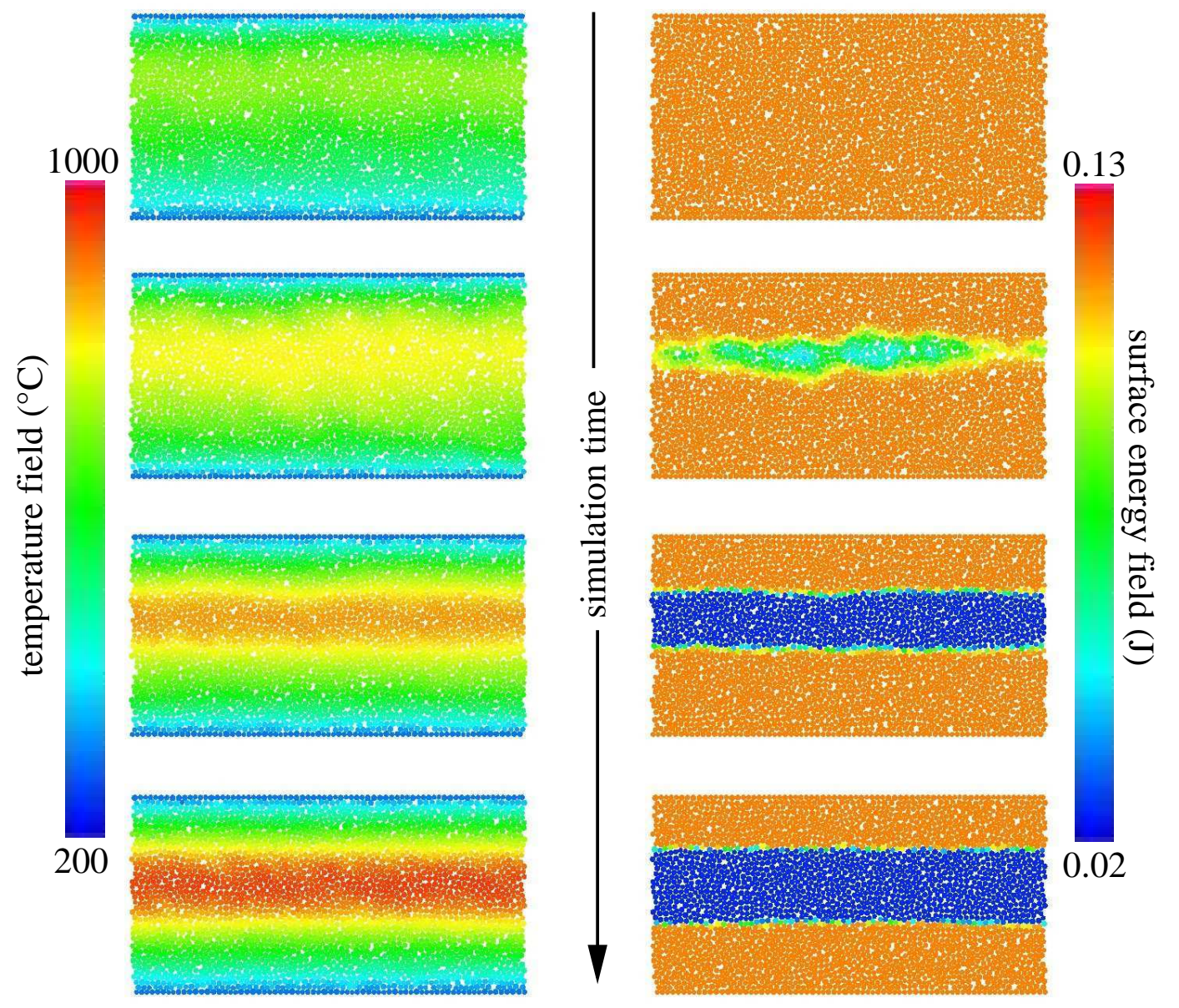

Figure 9: Evolution of temperature (left) and surface energy (right) during the simulation process

Friction transition occurs when the shear band appears in the sample. Thus, the creation of a small shear band is enough to ensure a reduction of the friction coefficient. The increase of shear band thickness has no impact on the friction coefficient. Secondly, the temperature within the sample decreases after localization occurs. As for the friction coefficient, the evolution 

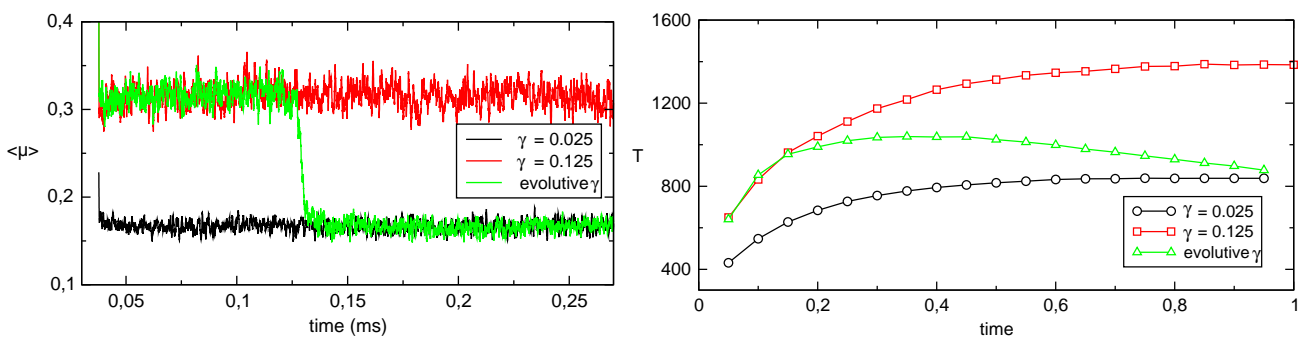

Figure 10: Evolution of the friction coefficient (left) and of the maximal temperature value (right) within the sample

of the temperature matches that of a simulation with strong cohesion which tends after localization to the evolution of the temperature of low cohesion. Nevertheless the transient phase of the temperature is longer than that of the friction coefficient. Such behaviour can be observed experimentally with carbon-carbon composites [18].

\section{Conclusion}

The present paper proposes a unified discrete element approach that can be used for the multi-physical modeling of discrete assemblies. This approach is well suited to modeling the multi-physical behaviour of third body flows. After several validations and comparisons (in static and dynamic situations), the thermal model allows linking velocities and temperature profiles during shearing processes: maximal temperature localizations and asymmetric temperature profiles can be directly correlated to the cohesion value used in a simulation for a given interaction law.

The physicochemical extension is illustrated with a basic an arbitrary law to describe the physicochemistry of the particles. The potential of the method 
is underlined as it allows reproducing the localization process resulting from the thermal properties of the sample. Nevertheless, the effective efficiency of this type of model will become more apparent when used in relation with a physical model, which is the objective of forthcoming works on real materials such as carbon-carbon composites.

\section{References}

[1] M. Godet. The third-body approach : a mechanical view of wear. Wear, 100:437-452, 1984.

[2] Y. Berthier, L. Vincent, and M. Godet. Velocity accommodation in fretting. Wear, 125(1-2):25-38, 1988.

[3] G. Lofficial, Y. Berthier, and M. Godet. Load carrying in slow reciprocating mechanisms. Tribology Series, 12:281-290, 1987.

[4] A. Jullien, M.-H. Meurisse, and Y. Berthier. Fractionated thin film lubrication. Tribology Series, 25:389-396, 1993.

[5] F. Ling and T.E. Simkins. Measurement of pointwise junction condition of temperatures at the interface of two bodies in sliding contact. ASME. J. Basic. Eng., 85:481-487, 1963.

[6] D. Majcherczak, P. Dufrenoy, and Y. Berthier. Tribological, thermal and mechanical coupling aspects of the dry sliding contact. Tribol. Int., 40(5):834-843, 2007.

[7] F.E. Kennedy. Thermal and thermomechanical effects of dry sling. Wear, 100:453-476, 1984. 
[8] S. Descartes, M. Renouf, N. Fillot, B. Gautier, A. Descamps, Y. Berthier, and P. Demanche. A new mechanical-electrical approach to the wheel-rail contact in wear. Wear, 265:1408-1416, 2008.

[9] P.A. Cundall. A computer model for simulating progressive large scale movements of blocky rock systems. In Proceedings of the symposium of the international society of rock mechanics, volume 1, pages 132-150, 1971.

[10] H.G. Elrod and D.E. Brewe. Numerical experiments with flows of elongated granules. Tribology Series, 21:219-226, 1992.

[11] A.A. Lubrecht and Y. Berthier. Granular lubrication; a simple model and trends. Tribology Series, 30:53-62, 1995.

[12] A. Ghaouti, M. Chaze, P. Dubujet, and F. Sidoroff. Particulate and granular simulation of the third body behaviour. Tribology Series, 31:355-365, 1996.

[13] N. Fillot, I. Iordanoff, and Y. Berthier. A granular dynamic model for the degradation of material. ASME J. Tribol., 126(3):606-614, 2004.

[14] M. Renouf and N. Fillot. Coupling electrical and mechanical effects in discrete element simulations. Int. J. Numer. Methods Eng., 74:238-254, 2008.

[15] D. Richard, I. Iordanoff, M. Renouf, and Y. Berthier. Thermal study of the dry sliding contact with third-body presence. ASME J. Tribol, 130(3):031404, 2008. 
[16] S. Descartes and Y. Berthier. Rheology and flows of solid third bodies: background and application to an mos1.6 coating. Wear, 252(7-8):546$556,2002$.

[17] J.R. Gomes, O.M. Silva, C.M. Silva, L.C. Pardini, and R.F. Silva. The effect of sliding speed and temperature on the tribological behaviour of carbon-carbonnext term composites. Wear, 249(3-4):240-245, 2001.

[18] H. Kasem, S. Bonnamy, Y. Berthier, P. Dufrénoy, and P. Jacquemard. Tribological, physicochemical and thermal study of the abrupt friction transition during carbon/carbon composite friction. Wear, 267(58):846-852, 2009.

[19] B.K. Yen, T. Ishihara, and I. Yamamoto. Influence of environment and temperature on "dusting" wear transitions of carbon-carbon composites. J. Mater. Sci., 32:681-686, 1997.

[20] P.A. Cundall and O.D.L. Strack. A discrete numerical model for granular assemblies. Geotechnique, 29(1):47-65, 1979.

[21] Y. Kishino. Disk model analysis of granular media. In Micromecanics of Granular Materials, pages 143-152, 1988. (eds. M. Satake and J.T. Jenkins),Elsevier.

[22] Y. Kishino, H. Akaizawa, and K. Kaneko. On the plastic flow of granular materials. In Y. Kishino, editor, Powder and grains, pages 199-202, 2001.

[23] M.P. Allen and D.J. Tildesley. Computer simulation of liquids. Oxford University Press, 1987. 
[24] J. J. Moreau. Unilateral contact and dry friction in finite freedom dynamics. In J.J. Moreau and eds. P.-D. Panagiotopoulos, editors, Non Smooth Mechanics and Applications, CISM Courses and Lectures, volume 302 (Springer-Verlag, Wien, New York), pages 1-82, 1988.

[25] R. W. Cottle, J. Pang, and R. E. Stone. The linear complementarity problem. Academic Press, Inc., Boston, MA, 1992.

[26] M. Jean. The non smooth contact dynamics method. Compt. Methods Appl. Math. Engrg., 177:235-257, 1999.

[27] B. Seve, I. Iordanoff, and Y. Berthier. A discrete solid third body model: Influence of the intergranular forces on the macroscopic behaviour. Tribology and Interface Engineering Series, 39:361-368, 2001.

[28] N. Fillot, I. Iordanoff, and Y. Berthier. Simulation of wear through a mass balance in a dry contact. ASME J. Tribol., 127(1):230-237, 2005.

[29] H.-P. Cao, F. Dubois, and M. Renouf. Hybrid discrete-finite element approach. application to tribological interfaces. In Proceeedings of the 4th International Conference on advanced computational methods in engineering, 2008.

[30] A. Kabir, M. Lovell, and C. F. Higgs III. Utilizing the explicit finite element method for studying granular flows. Tribology Letters, 29(2):8594, 2008.

[31] M. Jean, V. Acary, and Y. Monerie. Non-smooth contact dynamics approach of cohesive materials. Phil. Trans. R. Soc. Lond. A, 359:2497$2518,2001$. 
[32] G. Saussine, C. Cholet, F. Dubois, C. Bohatier, P.E. Gautier, and J.J. Moreau. Modelling ballast behaviour under dynamic loading. part 1: A 2 d polygonal discrete element method approach. Comput. Methods Appl. Mech. Engrg., 195(19-22):2841-2859, 2006.

[33] M. Renouf, D. Bonamy, F. Dubois, and P. Alart. Numerical simulation of two-dimensional steady granular flows in rotating drum: On surface flow rheology. Phys. Fluids, 17:103303, 2005.

[34] M. Renouf, F. Dubois, and P. Alart. A parallel version of the Non Smooth Contact Dynamics algorithm applied to the simulation of granular media. J. Comput. Appl. Math., 168:375-38, 2004.

[35] W.L. Vargas and J.J. McCarthy. Heat conduction in granular materials. AIChE Journal, 47(5):1052-1059, 2001.

[36] A. Helte. Radiative and conductive heate transfer in porous media: estimation of the effective thermal conductivity. J. Appl. Phys., 73(11):7167-7173, 1993.

[37] W.L. Vargas-Escobar. Discrete Modeling of Heat Conduction in Granular Media. PhD thesis, University of Pittsburgh, 2002.

[38] V.-D. Nguyen, C. Cogné, M. Guessasma, E. Bellenger, and J. Fortin. Discrete modeling of granular flow with thermal transfer: Application to the discharge of silos. Applied Thermal Engineering, 29(8-9):1846-1853, 2008.

[39] A. Bejan and A.D. Kraus. Heat Transfer Handbook. Hoboken, N.J.: J. Wiley \& sons, 2003. 
[40] D. Richard, I. Iordanoff, Y. Berthier, M. Renouf, and N. Fillot. Friction coefficient as a macroscopic view of local dissipation. ASME J. Tribol, 129(4):031404, 2007.

[41] K.L. Johnson, K. Kendall, and A.D. Roberts. Surface energy and the contact of elastic solids. Proc. R. Soc. London. A., 324:301-313, 1971.

[42] N.K. Myshkin, M.I. Petrokovets, and A.V. Kovalev. Tribology of polymers: adhesion, friction, wear, and mass-transfer. Tribol. Int., 38:910921, 2005.

[43] F.P. Bowden and D. Tabor. The friction and lubrication of solids. Oxford University Press, London, United Kingdom, 1958.

[44] T.S. Yun and J.C. Santamarina. Fundamental study of thermal conduction in dry soils. Granular Matter, 10:197-207, 2008. 